\title{
Die Beschwerdestelle für Probleme in der Altenpflege - ein Angebot der Stadt München
}

\author{
Kornelie Rahnema \\ Diplompsychologin, Leiterin der Beschwerdestelle für Probleme \\ in der Altenpflege der Stadt München
}

\section{Auftrag und Arbeitsweise}

Seit 1997 gibt es in München die Beschwerdestelle für Probleme in der Altenpflege als Anlaufstelle für pflegebedürftige alte Menschen, ihre Angehörigen, rechtliche Betreuer, andere Nahestehende oder Pflegekräfte. Wenn gewünscht wird deren Anonymität gewahrt.

Beschwerdeführende, die ihre Anliegen selbst weiterverfolgen möchten, werden dazu von den Mitarbeiterinnen (eine Psychologin als Leitung, vier Pflegefachkräfte mit Zusatzausbildungen) telefonisch oder persönlich fachkundig beraten und unterstützt.

Mit dem Einverständnis der Beschwerdeführenden können die Mitarbeiterinnen der Stelle aber auch in eigenen Prüfungen den vorgebrachten Beschwerden vor Ort selbst nachgehen. Die Träger(innen) der Pflegeeinrichtungen ermöglichen der Beschwerdestelle dazu auf freiwilliger Basis unangemeldete Besuche vor Ort. Die Beschwerdestelle ist im Vorfeld der gesetzlichen Kontrollbehörden (z.B. Heimaufsicht, Medizinischer Dienst der Krankenkassen) einzelfallbezogen tätig ohne Kompetenz zur Erteilung von Auflagen oder anderen Sanktionen. Nach Prüfung der Beschwerden und Abklärung und Einschätzung der Ursachen möglicher bestätigter Mängel wird mit allen Beteiligten nach bestmöglichen Lösungen und Verbesserungen für die Betroffenen gesucht. Dazu werden verbindliche Vereinbarungen getroffen und eine Wirkungsabfrage zum Ende der Bearbeitungszeit durchgeführt.

Die Einrichtungen und Träger werden jährlich zu den strukturellen Erkenntnissen über Schwachstellen beraten. Dem Oberbürgermeister und Stadtrat wird regelmäßig über die Arbeit der Beschwerdestelle berichtet, wenn möglich verbunden mit Vorschlägen für gezielte Verbesserungen. In verschiedenen Gremien und in der Öffentlichkeit vertritt die Beschwerdestelle die Interessen pflegebedürftiger alter Menschen.

Über sechs Jahre erhielt die Münchner Beschwerdestelle Modellfördermittel des Bundes und ist in ihrer Ausstattung und mit ihren Möglichkeiten eine einzigartige Einrichtung im Bundesgebiet.

\footnotetext{
Vielschichtigkeit der Problemlagen und Bearbeitungswege Alte und pflegebedürftige Menschen können sich wegen zunehmender Gebrechlichkeit oder geistiger Einschränkungen oft nicht mehr selbst vertreten. Sie werden zunehmend abhängig von anderen und dabei oft nicht mehr gefragt und als Person mit eigenen Wünschen und Bedürfnissen wahrgenommen. Die heute Hochaltrigen entstammen meist noch einer Genera-
}

tion, in der Anspruchslosigkeit und das geduldige Ertragen von Problemlagen als Werte angesehen wurden. Insbesondere die alten Frauen haben wenig gelernt, sich für ihre Rechte einzusetzen.

Andere Menschen, ihre Angehörigen oder auch rechtliche Betreuer(innen), sprechen oft für die pflegebedürftigen alten Menschen und wenden sich an die Beschwerdestelle. Sie haben eigene Blickwinkel, unterschiedliche Motivationen und bewegen sich nicht selten in Spannungsfeldern zwischen Überfürsorglichkeit, Berührungsängsten, Schuldgefühlen und schwierigen biografischen Erfahrungen. Gerade pflegende Angehörige, auch hier überwiegend die Ehefrauen, Töchter und Schwiegertöchter, geraten nicht selten an die Grenzen der eigenen Ressourcen.

Die Bearbeitung von Beschwerden erfordert daher große Einfühlsamkeit in die emotionale Verfassung der Beschwerdeführenden, bevor eine Klärung der Sachebene erfolgen kann. Dort, wo es möglich ist, bemühen sich die Mitarbeiterinnen der Beschwerdestelle, durch Besuche bei den Betroffenen zu Hause oder in Pflegeheimen deren Wünsche, Bedürfnisse und Interessen zu erfahren und ihre Betreuungssituation selbst fachkundig einzuschätzen.

Ziel der weiteren Beschwerdebearbeitung ist es in erster Linie, dort, wo Mängel und Versäumnisse erkannt werden, alle Beteiligten für gemeinsame Veränderungsprozesse zur Verbesserung in der Versorgungssituation für Betroffene zu gewinnen. Nachrangig werden dann andere Themen geklärt und bearbeitet. Es geht in diesen Veränderungsprozessen nicht um Schuldzuweisungen und Anklagen, sondern um die Verbesserung der Lebensqualität pflegebedürftiger alter Menschen. Dies wird erreicht durch die Förderung einer offenen Fehlerkultur mit Respekt für die Blickwinkel aller Beteiligter. Konkrete Vereinbarungen zur Verankerung von Pflegefachlichkeit im Alltag oder zur Verbesserung von Abstimmung und Informationsfluss gehören genauso zur Arbeit der Beschwerdestelle wie die Vermittlung bei zwischenmenschlichen Konflikten. Zum Abschluss der bis zu mehrere Monate dauernden Begleitung erfolgt eine Wirkungsabfrage und -einschätzung aus den Blickwinkeln aller Beteiligter.

\section{Erkenntnisse aus der Bearbeitung von Beschwerden}

Die Grundversorgung pflegebedürftiger alter Menschen wird über die Sozialgesetzgebung und die Rahmenbedingungen im Wesentlichen ermöglicht. Defizite bestehen seit Jahren bei der Betreuung demenziell Erkrankter, bei Prophylaxe und Rehabilitation, bei der Verwirklichung von mobilisierender und aktivierender Pflege, bei ärztlicher und palliativer Betreuung. Für eine individuelle, bedarfsorientierte, ganzheitliche und zielgerichtete Betreuung und Pflege reichen die derzeitigen Rahmenbedingungen nicht aus. 
Mängel und bestätigte Beschwerden gibt es sowohl auf Leitungsebene wie in der konkreten Pflege. Fachliche Defizite im Umgang mit Medikamenten, unzureichende Beachtung von Schmerzen, Mängel bei der Hygiene, unzureichende Planung pflegerischen Handelns sind Schwerpunkte auf der Pflegeebene. Die Leitungen sind verantwortlich für Mängel bei der Essens- und Wohnqualität, für Probleme bei Einsatzplanung und Anleitung der Pflegekräfte, für Fehler in der Vertragsgestaltung und Abrechnung. Häufig liegen Schwierigkeiten auch auf der Beziehungsebene, wenn Information, Kommunikation und Abstimmung zwischen den Beteiligten (Angehörige, Betroffene, Pflegekräfte, Einsatzleitungen, Ärzt(inn)e(n) u.a.) nicht ausreichend funktionieren. Unterbesetzung, Arbeitsbelastung, Betriebsblindheit, Unachtsamkeit, fehlende Anleitung und Kontrolle sind dafür genauso ursächlich wie unzureichende Fachlichkeit bei Planung der Pflege und Verknüpfung von Beobachtungen und Wissen mit gezielten Maßnahmen. Die Einstellung von Träger(inne)n und Leitungen zu Beschwerden, ihr Qualitäts- und Personalmanagement, ihre Bemühungen um Personalgewinnung oder Aus- und Weiterbildung haben direkten Einfluss auf die betriebsinterne Fehlerkultur und Qualitätsentwicklung.

\section{Die Pflege - ein Frauenthema}

Die Beschwerdestelle beobachtete in den Jahren ihrer Tätigkeit aufmerksam die geschlechtsspezifischen Unterschiede in den Lebensbedingungen alter Menschen, in ihrem Zugang zu Leistungen aus der Pflegeversicherung ebenso wie in den Bedingungen der pflegenden Angehörigen und der Pflegekräfte. Selten werden die unterschiedlichen Voraussetzungen und Bedingungen von Frauen und Männern in der Altenpflege wahrgenommen oder gar berücksichtigt. Eine geschlechterdifferenzierte Sicht und Ausgestaltung von Angeboten und Leistungen ist noch zu wenig präsent.

Die Lebenssituation der überwiegenden Mehrheit von Frauen im Alter unterscheidet sich hinsichtlich Einkommen, Wohnsituation, Mobilität und Gesundheitszustand deutlich von der der Männer. Männer und Frauen haben unterschiedliche Unterstützungssysteme. Entlastungsmöglichkeiten sind oft abhängig von der unterschiedlichen Einkommenssituation. Alte Männer erhalten häufiger familiäre Hilfe durch Ehefrauen oder andere - meist weibliche - Angehörige. Frauen leben im Alter häufiger allein und erfahren notwendige Hilfe eher durch Freundinnen oder Nachbarinnen. Rollenmuster und frühere Arbeitsteilung erschweren es alten Frauen nicht selten, eigene Rechte zu erkennen und durchzusetzen, sei es bei Behördengängen, im Schriftverkehr oder in Geschäften mit Banken und Versicherungen, wenn dafür vorher alleine der Partner zuständig war.

Rund zwei Drittel der Pflegebedürftigen sind weiblich. Es gibt derzeit keine geschlechterdifferenzierten Begutachtungsrichtlinien bei der Prüfung der Zugangsvoraussetzungen für Leistungen aus der Pflegeversicherung durch den Medizinischen Dienst der Krankenversicherung (MDK).

Pflegende Angehörige sind in der Mehrzahl Frauen, jede zweite Pflegeperson ist selbst schon über 55 Jahre alt. Sie sind oft über viele Jahre einer dauernden hohen physischen wie psychischen Belastung ausgesetzt. Besonders Frauen brauchen dabei eine Unterstützung zur Selbstpflege, da sie in ihrer Rolle meist den Auftrag vermittelt bekamen und es erlernt haben, für andere zu sorgen.

Durchschnittlich 86 Prozent der Pflegekräfte in ambulanten Diensten und stationären Einrichtungen sind weiblich, während die Leitungsebene überwiegend männlich besetzt ist. Doppelbelastungen von Familie und Beruf, schwierige Arbeitsbedingungen, geringe Bezahlung und wenig gesellschaftliche Wertschätzung prägen die Tätigkeit dieser Frauen.

\section{Handlungsbedarf}

Die Altenpflege krankt grundsätzlich an den längst überfälligen Reformen der Rahmenbedingungen. Das System der Pflegeversicherung baut ausdrücklich auf eigenverantwortlichen und familiären Versorgungssystemen auf, die vorwiegend von Frauen getragen werden. Die Entlastung und Ergänzung der familiären Pflege bedarf großer Aufmerksamkeit. Die Personalnot in der professionellen Pflege wird zunehmen und die notwendige fachkompetente Versorgung pflegebedürftiger alter Menschen erschweren. Mängel in der Qualität der Dienstleistungen sind die Folge und bedürfen einer Bereitschaft aller Beteiligter zu einer konstruktiven Fehlerkultur. Um in der Altenpflege geschlechtergerecht zu planen und zu handeln brauchen die Verantwortlichen entsprechendes Wissen und Kompetenz. Noch gibt es dazu wenige wissenschaftlich fundierte Erkenntnisse.

Die Altenpflege ist ein gesellschaftlicher Bereich, in dem Benachteiligungen von Frauen als Pflegebedürftige, als pflegende Angehörige wie als professionelle Pflegekräfte besonders deutlich sind. Daher muss Pflegepolitik immer auch Frauenpolitik sein.

\section{Literaturhinweise}

Bundesministerium für Familie, Senioren, Frauen und Jugend: Charta der Rechte hilfe- und pflegebedürftiger Menschen, 10. Auflage, Berlin 2010

Landeshauptstadt München: Altenhilfe: Geschlechter gerecht? Dokumentation der Fachtagung am 10. Oktober 2006, erhältlich im Internet: <http://www.muenchen.de/cms/prod2/mde/_de/rubriken/ Rathaus/40_dir/altenpfl/o8_veroeff/innen2.pdf> (Zugriff: 31.3.2011)

Beschwerdestelle für Probleme in der Altenpflege der Landeshauptstadt München: Bericht über die Tätigkeit in den Jahren 2007 und 2008, erhältlich im Internet: <http://www.muenchen.de/cms/prod2/ mde/_de/rubriken/Rathaus/40_dir/altenpfl/o8_veroeff/ 2009_07_27_Druckvorlage_Bericht2009.pdf> (Zügriff: 31.3.2011) 